\title{
Low-Thrust Many-Revolution Trajectory Optimization via Differential Dynamic Programming and a Sundman Transformation
}

Jonathan Aziz, Jeffrey Parker, Daniel Scheeres

University of Colorado Boulder

Jacob Englander

NASA Goddard Space Flight Center 


\section{Planetary}

- Long transfer durations with short orbital periods span many revolutions

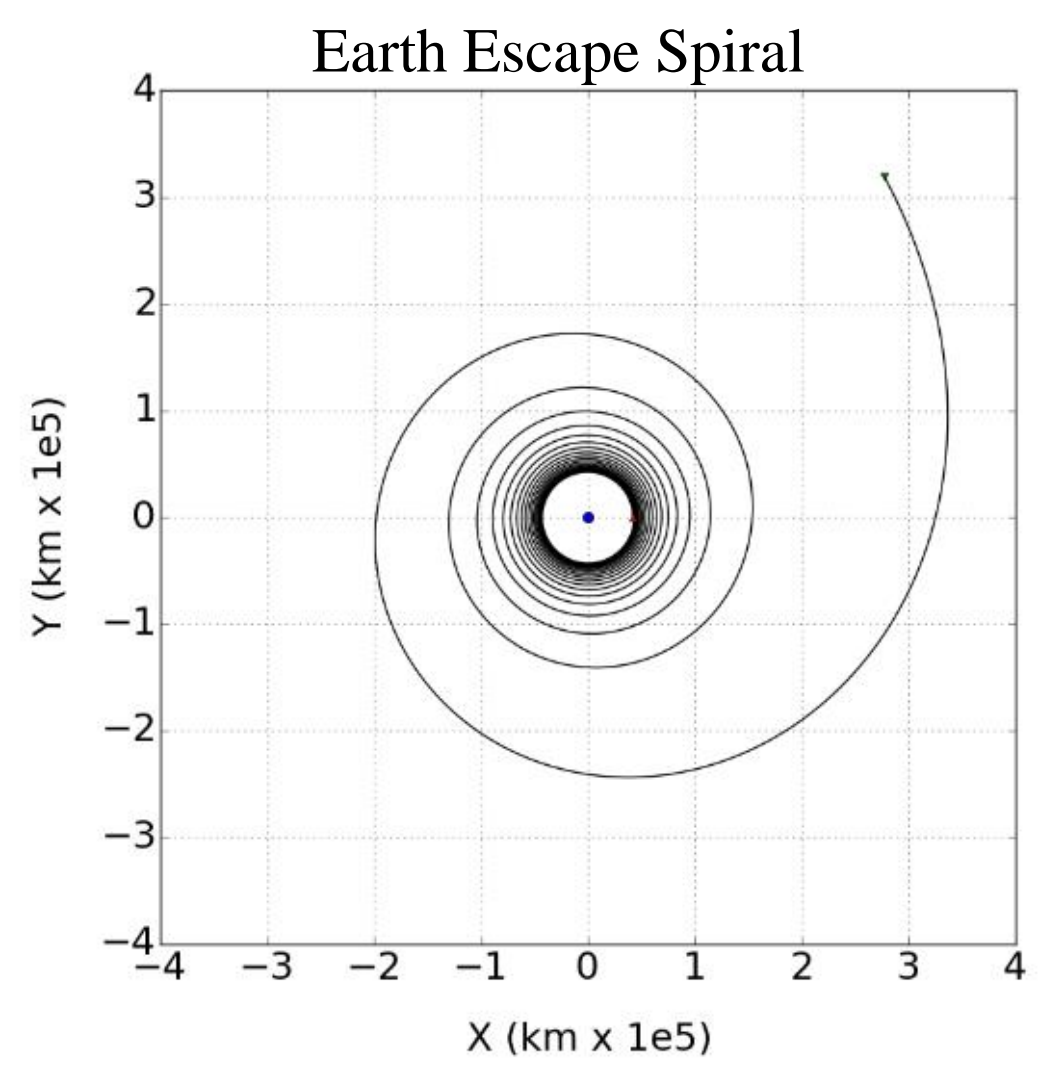

Number of 'revs': 10s, 100s, 1000s

\section{Interplanetary}

- Slow dynamics compared to control schedule

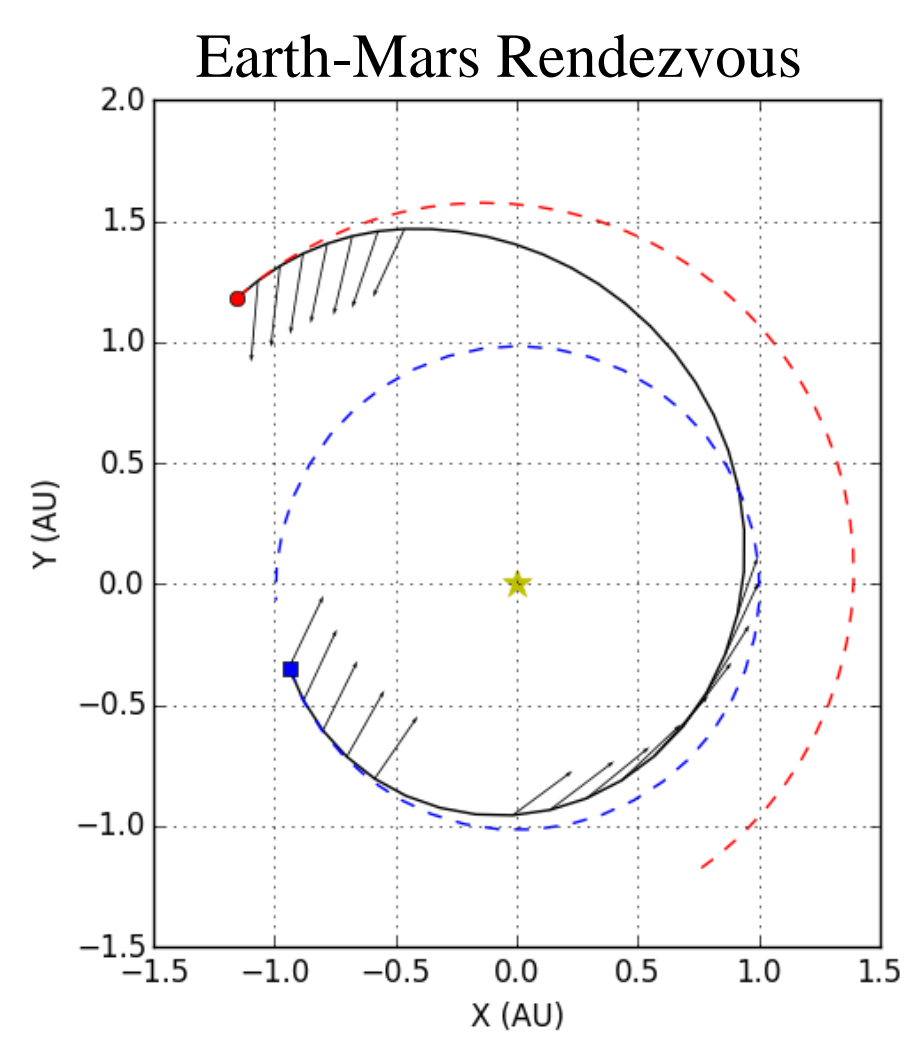

$<1,1-10,10$ s 


\section{Indirect}

optimal control theory, apply Euler-Lagrange theorem and solve two point boundary value problem (TPBVP)

\section{Control Laws}

set a rule for spacecraft steering - a suboptimal policy that is acceptable by the mission designer

\section{Direct}

transcribe the trajectory optimization into a parameter optimization problem
Edelbaum
Alfano
Kéchichian

\author{
Kluever \\ Chang \\ Petropolous
}

Betts

Whiffen

Lantoine 


\section{- Hybrid Differential Dynamic Programming}

- introduced by Lantoine and Russell

- sequence of control updates that minimize quadratic model of cost-to-go

- map derivatives along trajectory with state transition matrix and tensor

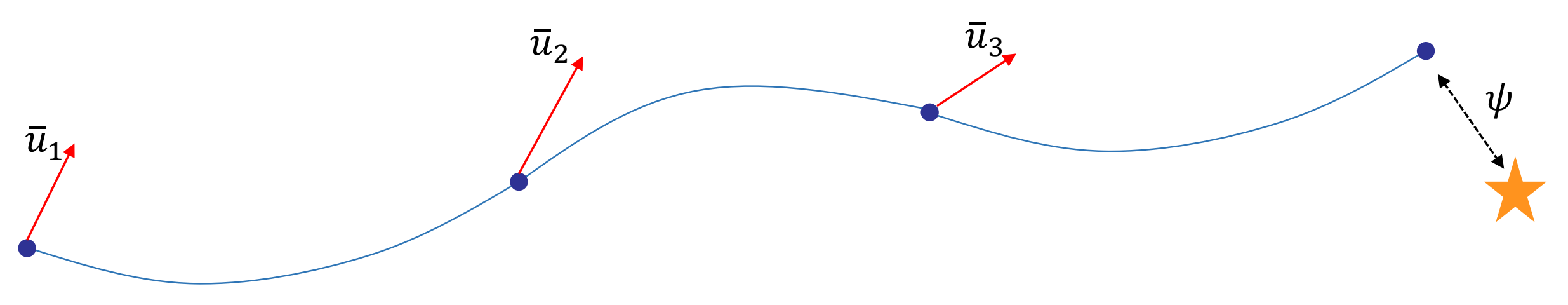




\section{- Hybrid Differential Dynamic Programming}

- introduced by Lantoine and Russell

- sequence of control updates that minimize quadratic model of cost-to-go

- map derivatives along trajectory with state transition matrix and tensor

Quadratic model

from derivatives of $\psi$

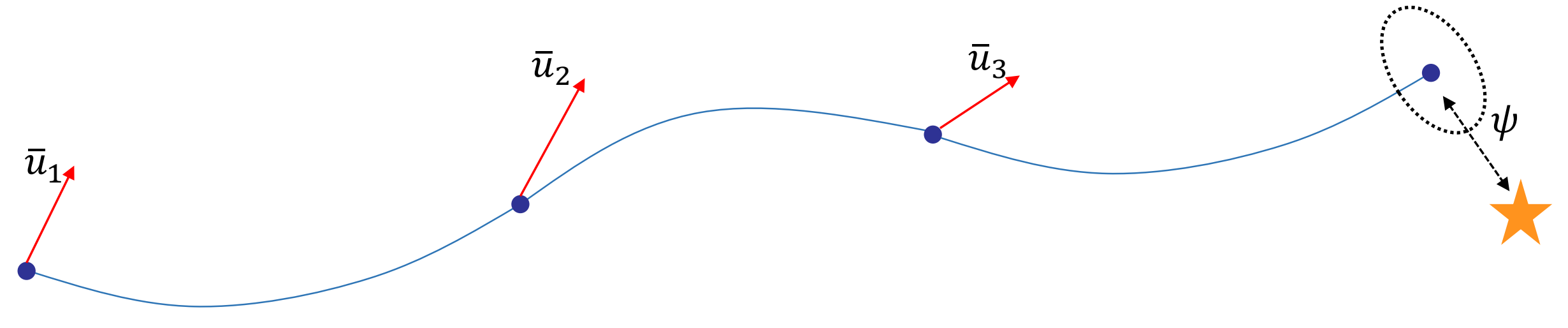




\section{- Hybrid Differential Dynamic Programming}

- introduced by Lantoine and Russell

- sequence of control updates that minimize quadratic model of cost-to-go

- map derivatives along trajectory with state transition matrix and tensor

Quadratic model from derivatives of $\psi$

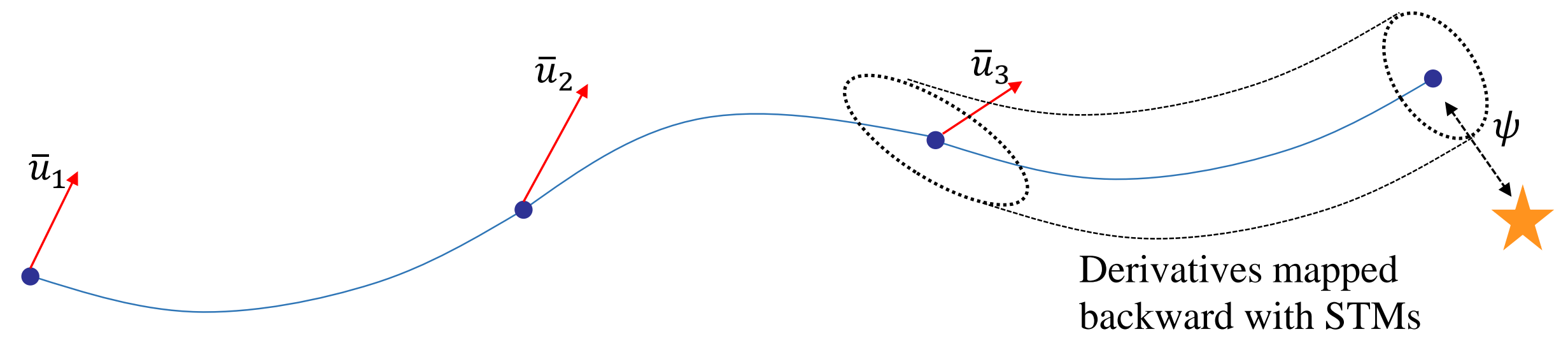




\section{- Hybrid Differential Dynamic Programming}

- introduced by Lantoine and Russell

- sequence of control updates that minimize quadratic model of cost-to-go

- map derivatives along trajectory with state transition matrix and tensor

Quadratic model

Minimize local model

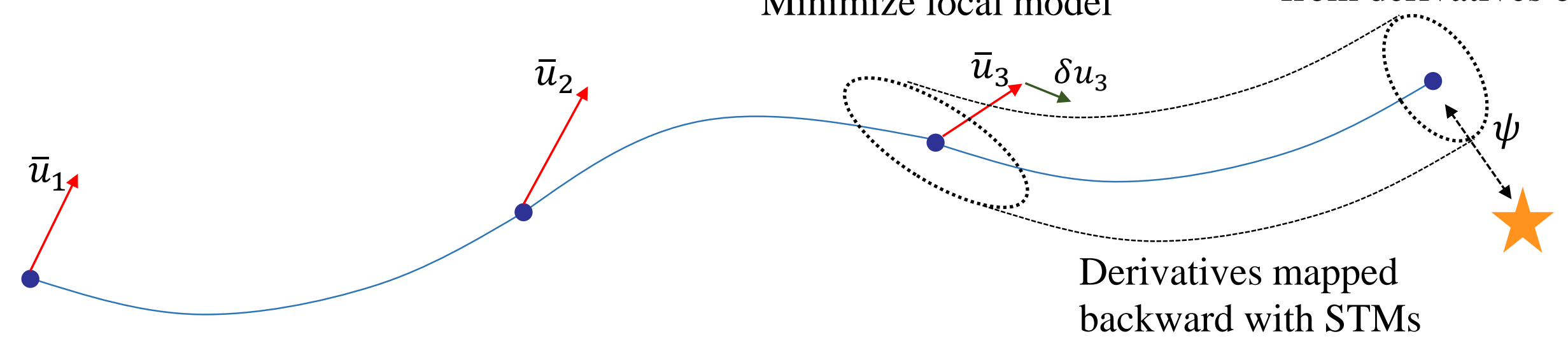




\section{- Hybrid Differential Dynamic Programming}

- introduced by Lantoine and Russell

- sequence of control updates that minimize quadratic model of cost-to-go

- map derivatives along trajectory with state transition matrix and tensor

Quadratic model

Minimize local model

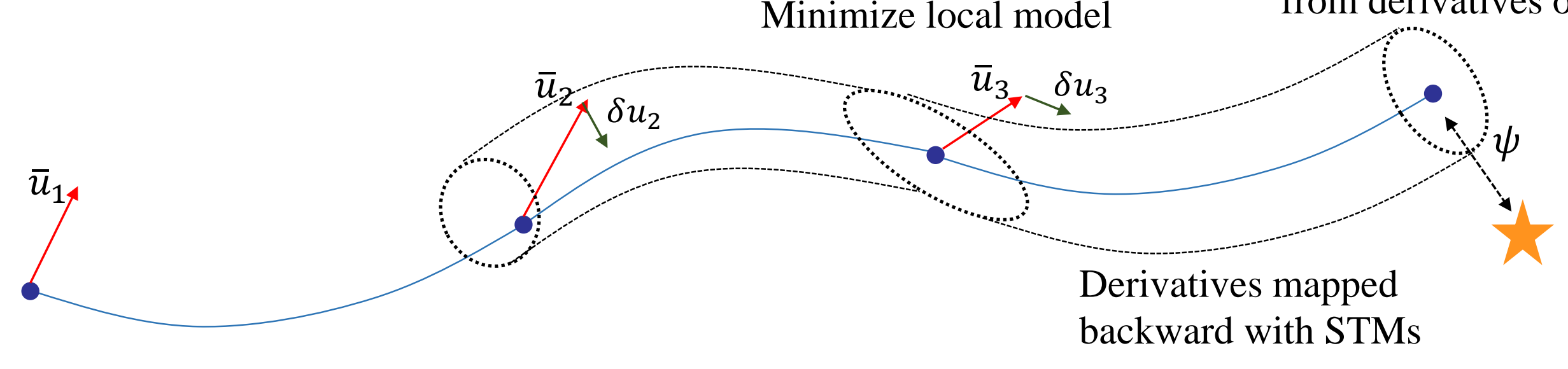




\section{- Hybrid Differential Dynamic Programming}

- introduced by Lantoine and Russell

- sequence of control updates that minimize quadratic model of cost-to-go

- map derivatives along trajectory with state transition matrix and tensor

Quadratic model

Minimize local model

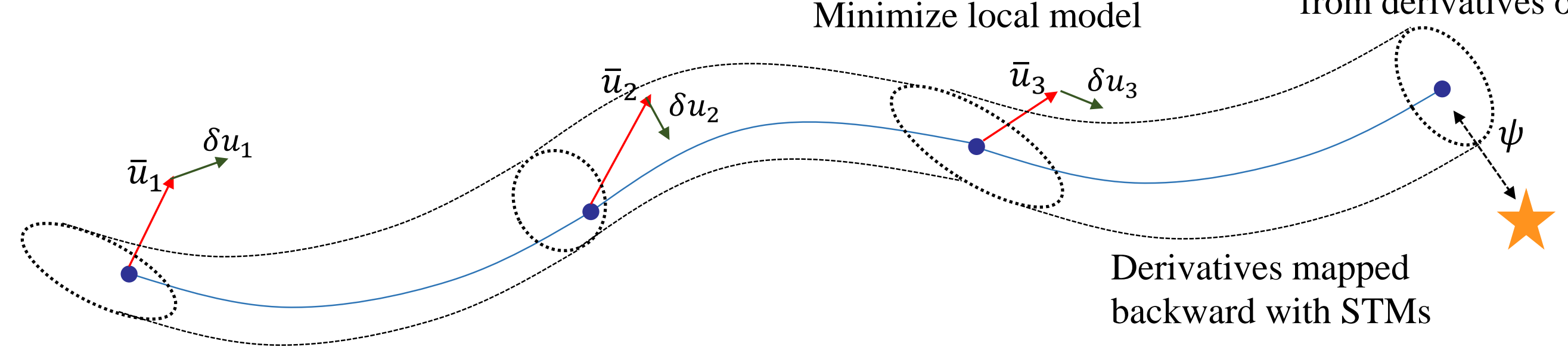

- Forward pass: evaluate $\bar{u}+\delta u$ in equations of motion

- Backward sweep: compute each $\delta u_{k}$ 


\section{- Hybrid Differential Dynamic Programming}

- introduced by Lantoine and Russell

- sequence of control updates that minimize quadratic model of cost-to-go

- map derivatives along trajectory with state transition matrix and tensor

Quadratic model

Minimize local model

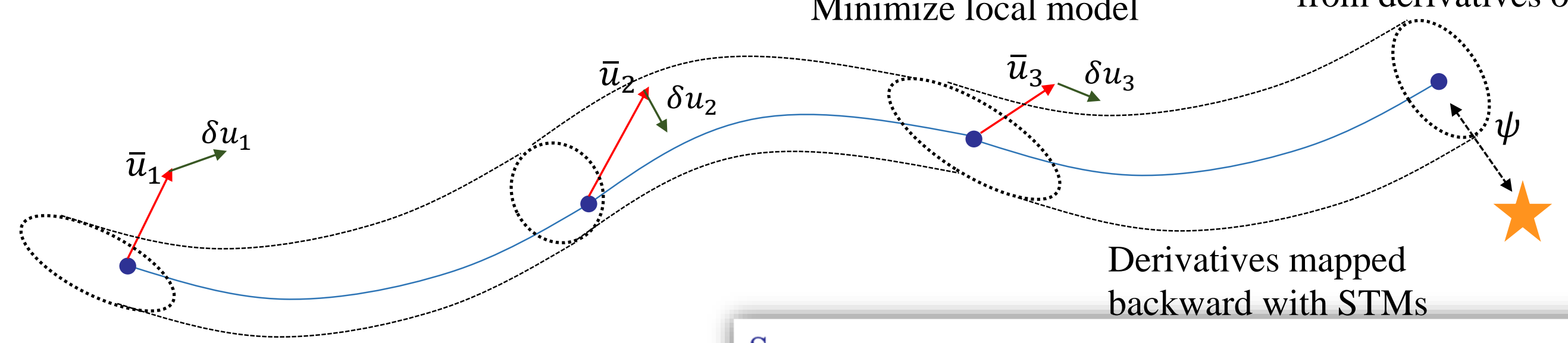

See:

- Forward pass: evaluate $\bar{u}+\delta u$ i Gregory Lantoine and Ryan P. Russell. A hybrid differential

- Backward sweep: compute each dynamic programming algorithm

for constrained optimal control problems. part 1: Theory. Journal of Optimization Theory and Applications, 154(2):382-417, 2012. 
- Change independent variable from time to a function of orbital radius

$$
d t=c_{n} r^{n} d \tau
$$

- Can choose $n, c_{n}$, so that $\tau$ is an orbit angle

\begin{tabular}{c|c|c} 
Eccentric Anomaly & Mean Anomaly & True Anomaly \\
\hline$d t=\sqrt{\frac{a}{\mu}} r d E$ & $d t=\sqrt{\frac{a^{3}}{\mu}} d M$ & $d t=\frac{r^{2}}{h} d v$
\end{tabular}

- Equations of motion become $x^{\prime}=\dot{x} c_{n} r^{n}$

- Discretize in $\tau$

- Specify $\tau_{0}, \tau_{f}$, rather than $t_{0}, t_{f}$

- i.e. specify number of revolutions
- Track time in the state vector

$$
x=\left[\begin{array}{c}
t \\
x \\
y \\
z \\
\vdots
\end{array}\right], \quad x^{\prime}=\left[\begin{array}{c}
1 \\
\dot{x} \\
\dot{y} \\
\dot{z} \\
\vdots
\end{array}\right] c_{n} r^{n}
$$


- Choose $\tau=E$, the eccentric anomaly

- Minimum fuel GTO to GEO in 450.5 revs

- 2-body dynamics

- 135,150 variables

- 54 minutes
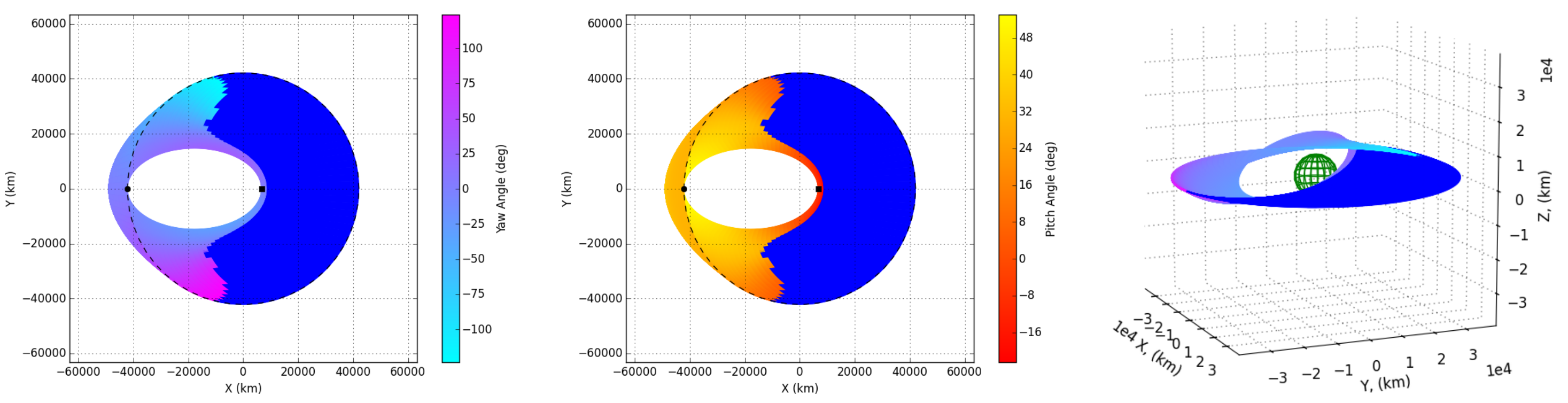
- Choose $\tau=E$, the eccentric anomaly

- Minimum fuel GTO to GEO in 450.5 revs

- 2-body dynamics $+J_{2}$

- 135,150 variables

- 61 minutes
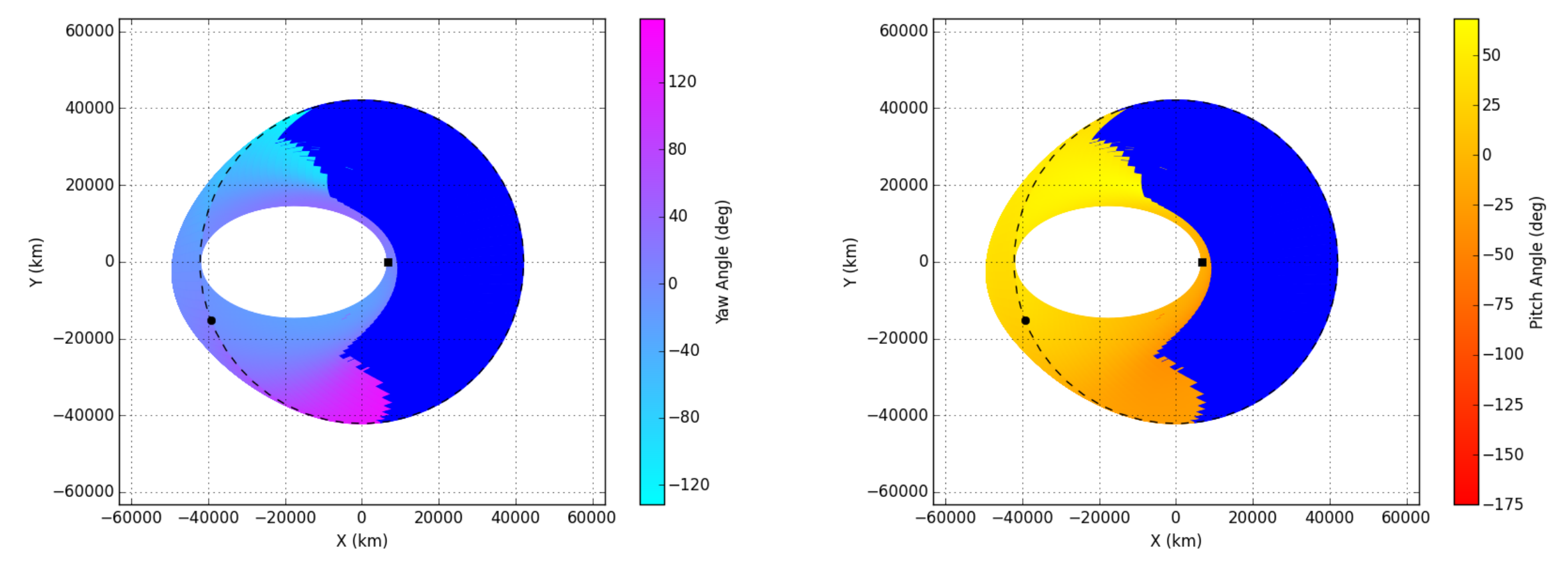
- Choose $\tau=E$, the eccentric anomaly

- Minimum fuel GTO to GEO in 450.5 revs

- 2-body dynamics $+J_{2}+$ lunar gravity

- 135,150 variables

- 107 minutes
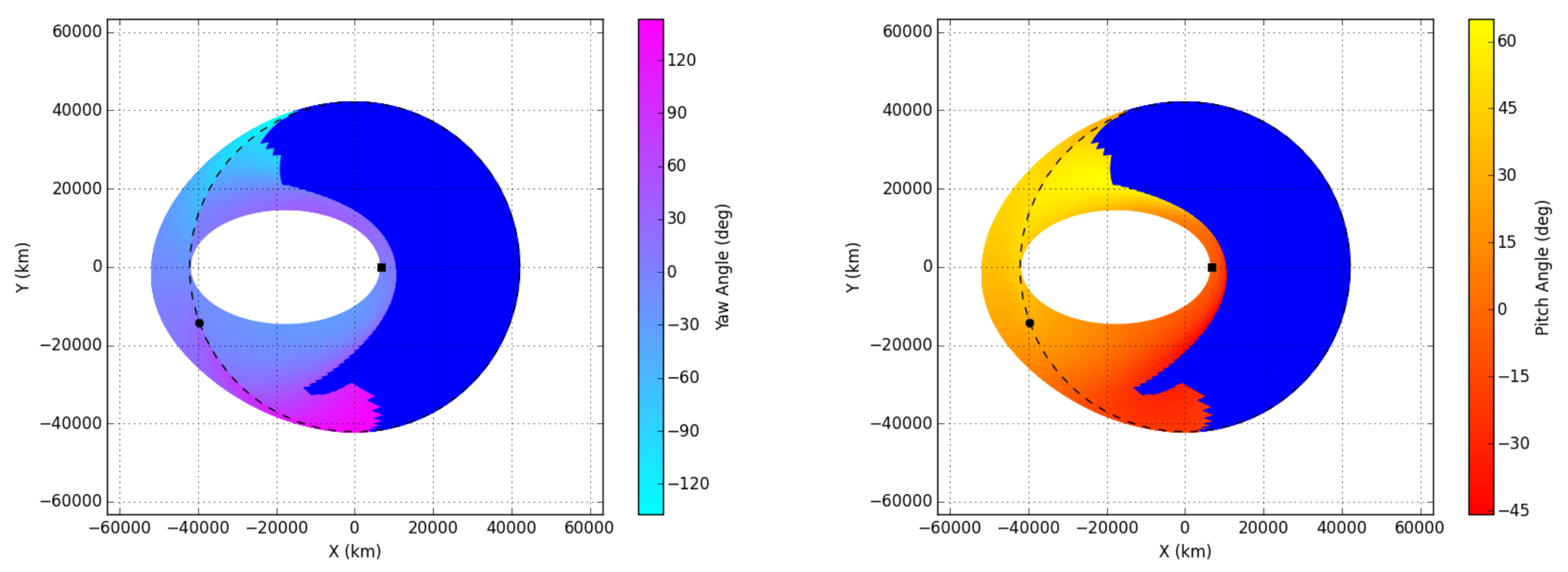
- Choose $\tau=E$, the eccentric anomaly

- Minimum fuel GTO to GEO in 1000.5 revs

- 2-body dynamics $+J_{2}+$ lunar gravity

- 300,150 variables

- 1359 minutes
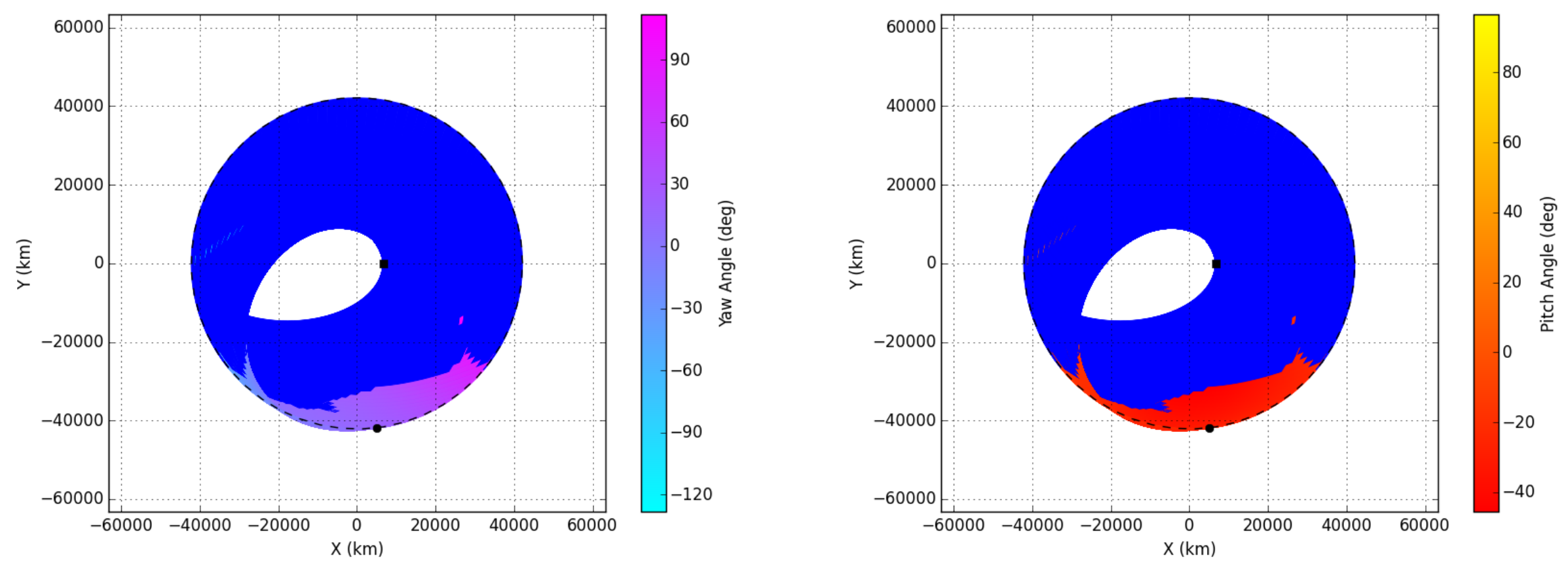


\section{Backup Slides}


Table 2.1: Summary of GTO to GEO Results.

\begin{tabular}{|c|c|c|c|c|c|c|}
\hline Perturbations & $N_{\text {rev }}$ & Iterations & Runtime (minutes) & $m_{f}(\mathrm{~kg})$ & $t_{f}$ (days) & $\theta(\mathrm{deg})$ \\
\hline \hline None & 450.5 & 86 & 54 & 1759.1754 & 315.75 & 180.0 \\
\hline$J_{2}$ & 450.5 & 111 & 61 & 1737.1949 & 342.13 & 199.8728 \\
\hline$J_{2}$ and Lunar Gravity & 450.5 & 136 & 107 & 1745.3012 & 322.63 & 201.0805 \\
\hline$J_{2}$ and Lunar Gravity & 1000.5 & 913 & 1359 & 1784.3632 & 558.86 & 276.7209 \\
\hline None (Eclipse Model) & 450.5 & 93 & 70 & 1751.4223 & 325.48 & 180.0 \\
\hline None (MEE) & 450.5 & 59 & 15 & 1758.7230 & 318.96 & 180.0 \\
\hline
\end{tabular}


500 rev orbit lowering at Mars
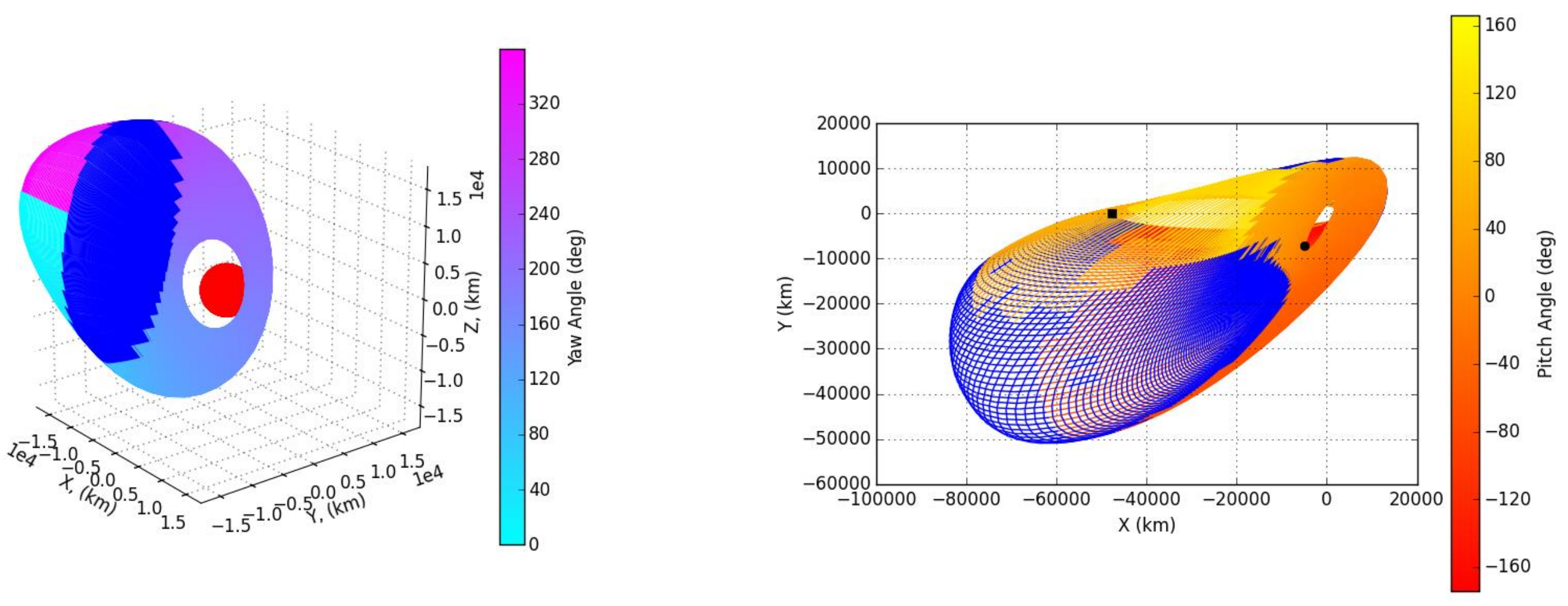

and with $\Delta \Omega=60^{\circ}$ 
- Feedback control laws for $\delta u, \delta \lambda, \delta w$ are unconstrained

- Likely to step beyond validity of quadratic expansion

- Require invertible, positive definite Hessians (negative definite for $J_{\lambda \lambda}$ )

- Trust-region quadratic subproblem (TRQP):

$$
\begin{aligned}
& \min _{\delta \boldsymbol{u}_{k}}\left[J_{u, k}^{T} \delta \boldsymbol{u}_{k}+\frac{1}{2} \delta \boldsymbol{u}_{k}^{T} J_{u u, k} \delta \boldsymbol{u}_{k}\right] \\
& \text { s.t. }\left\|D \delta \boldsymbol{u}_{k}\right\| \leqslant \Delta
\end{aligned}
$$

- Acceptance of an iterate:

$$
\rho=\frac{\delta J}{E R_{0,0}} \quad \Delta_{p+1}= \begin{cases}\min \left((1+\kappa) \Delta_{p}, \Delta_{\max }\right), & \text { if } \rho \in\left[1-\epsilon_{1}, 1+\epsilon_{1}\right] \\ \max \left((1-\kappa) \Delta_{p}, \Delta_{\min }\right), & \text { otherwise }\end{cases}
$$




$$
\begin{aligned}
& \boldsymbol{X}=\left[\begin{array}{lllllllllll}
t & x & y & z & \dot{x} & \dot{y} & \dot{z} & m & T & \alpha & \beta
\end{array}\right]^{T} \\
& {\left[\begin{array}{lll}
\hat{\boldsymbol{r}} & \hat{\boldsymbol{s}} & \hat{\boldsymbol{w}}
\end{array}\right]=\left[\begin{array}{lll}
\frac{\boldsymbol{r}}{r} & \frac{(\boldsymbol{r} \times \boldsymbol{v}) \times \boldsymbol{r}}{\|(\boldsymbol{r} \times \boldsymbol{v}) \times \boldsymbol{r}\|} & \frac{\boldsymbol{r} \times \boldsymbol{v}}{\|\boldsymbol{r} \times \boldsymbol{v}\|}
\end{array}\right] .} \\
& {\left[\begin{array}{c}
T_{r} \\
T_{s} \\
T_{w}
\end{array}\right]=\left[\begin{array}{c}
T \sin \alpha \cos \beta \\
T \cos \alpha \cos \beta \\
T \sin \beta
\end{array}\right],\left[\begin{array}{c}
T_{x} \\
T_{y} \\
T_{z}
\end{array}\right]=\left[\begin{array}{lll}
\hat{\boldsymbol{r}} & \hat{\boldsymbol{s}} & \hat{\boldsymbol{w}}
\end{array}\right]\left[\begin{array}{c}
T_{r} \\
T_{s} \\
T_{w}
\end{array}\right],}
\end{aligned}
$$

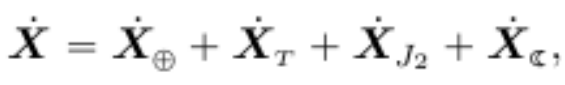

$$
\begin{aligned}
& \dot{\boldsymbol{X}}_{\oplus}=\left[\begin{array}{lllllllllll}
1 & \dot{x} & \dot{y} & \dot{z} & -\frac{\mu_{\oplus}}{r^{3}} x & -\frac{\mu_{\oplus}}{r^{3}} y & -\frac{\mu_{\oplus}}{r^{3}} & 0 & 0 & 0 & 0
\end{array}\right]^{T}, \\
& \dot{\boldsymbol{X}}_{T}=\left[\begin{array}{lllllllllll}
0 & 0 & 0 & 0 & \frac{T_{x}}{m} & \frac{T_{y}}{m} & \frac{T_{z}}{m} & -\frac{T}{I_{s p} g_{0}} & 0 & 0 & 0
\end{array}\right]^{T}, \\
& \dot{\boldsymbol{X}}_{J_{2}}=-\frac{3 J_{2} \mu_{\oplus} R_{\oplus^{2}}}{2 r^{5}}\left[\begin{array}{lllllllllll}
0 & 0 & 0 & 0 & x\left(1-5 \frac{z^{2}}{r^{2}}\right) & y\left(1-5 \frac{z^{2}}{r^{2}}\right) & z\left(3-5 \frac{z^{2}}{r^{2}}\right) & 0 & 0 & 0 & 0
\end{array}\right]^{T},
\end{aligned}
$$

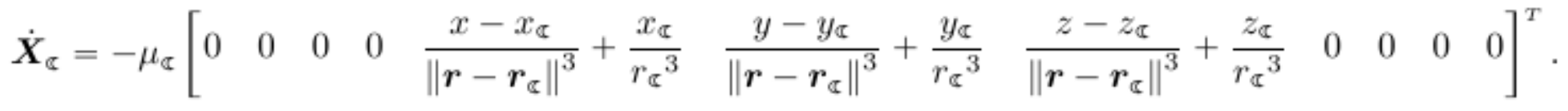




$$
\stackrel{\circ}{\boldsymbol{X}}=\left(\dot{\boldsymbol{X}}_{\oplus}+\dot{\boldsymbol{X}}_{T}+\dot{\boldsymbol{X}}_{J_{2}}+\dot{\boldsymbol{X}}_{\mathbb{c}}\right) \sqrt{a / \mu_{\oplus}} r
$$

$$
\begin{gathered}
A^{i, j}=\frac{\partial \dot{\boldsymbol{X}}^{i}}{\partial \boldsymbol{X}^{j}}, \\
A^{i, j k}=\frac{\partial^{2} \dot{\boldsymbol{X}}^{i}}{\partial \boldsymbol{X}^{j} \partial \boldsymbol{X}^{k}},
\end{gathered}
$$

$$
\Lambda^{i, j}=\frac{\partial \stackrel{\circ}{\boldsymbol{X}}^{i}}{\partial \boldsymbol{X}^{j}}
$$$$
\Lambda^{i, j k}=\frac{\partial^{2} \dot{\boldsymbol{X}}^{i}}{\partial \boldsymbol{X}^{j} \partial \boldsymbol{X}^{k}}
$$$$
\eta=d t / d \tau=c_{n} r^{n}
$$$$
\eta_{X}{ }^{i}=\frac{\partial \eta}{\partial \boldsymbol{X}^{i}}
$$$$
\eta_{X X}{ }^{i, j}=\frac{\partial^{2} \eta}{\partial \boldsymbol{X}^{i} \partial \boldsymbol{X}^{j}}
$$

$$
\Lambda^{i, j}=A^{i, j} \eta+\dot{X}^{i} \eta_{x}^{j}
$$$$
\Lambda^{i, j k}=A^{i, j k} \eta+A^{i, j} \eta_{X}{ }^{k}+A^{i, k} \eta_{X}^{j}+\dot{X}^{i} \eta_{X X}{ }^{j, k}
$$

$$
\begin{gathered}
\Phi^{i, a}\left(t_{k}, t_{k+1}\right)=\frac{\partial \boldsymbol{X}_{k+1}^{i}}{\partial \boldsymbol{X}_{k}^{a}}=\boldsymbol{F}_{X, k}^{i, a} \\
\Phi^{i, a b}\left(t_{k}, t_{k+1}\right)=\frac{\partial^{2} \boldsymbol{X}_{k+1}^{i}}{\partial \boldsymbol{X}_{k}^{a} \partial \boldsymbol{X}_{k}^{b}}=\boldsymbol{F}_{X X, k}^{i, a b} \\
\dot{\Phi}^{i, a}=A^{i, \gamma_{1}} \Phi^{\gamma_{1}, a} \\
\dot{\Phi}^{i, a b}=A^{i, \gamma_{1}} \Phi^{\gamma_{1}, a b}+A^{i, \gamma_{1} \gamma_{2}} \Phi^{\gamma_{1}, a} \Phi^{\gamma_{2}, b}
\end{gathered}
$$

(21b) $\quad \stackrel{\circ}{\Phi}^{i, j k}=\Lambda^{i, a} \Phi^{a, j k}+\Lambda^{i, a b} \Phi^{a, j} \Phi^{b, k}$ 